\title{
Anticipatory Controller Design for Dual-stator Toroidal Motor with Hybrid Excitation
}

\author{
Xin Liu, Ling Nie \\ Tianjin key Laboratory of modern electromechanical equipment technology, \\ Tianjin polytechnic university, Tianjin, 300387, China \\ email: liuxin@tjpu.edu.cn
}

\section{Keywords: Toroidal Motor; Speed Control; Controller Design}

\begin{abstract}
Dual-stator toroidal motor is a new style of motor, it integrates power and drive. The electromechanical coupled model is built based on the operating principle of the motor. The transfer function of the motor system is derived. In consideration of the response characteristic of toroidal motor, a speed feedback control scheme is presented and a anticipatory controller is designed based on performance index. The step response of the closed-loop speed control system of the motor is simulated. The results justify the validity of the controller, and useful for designing controller and practical application for the novel motor.
\end{abstract}

\section{Introduction}

Dual-stator toroidal motor with hybrid excitation which integrates power and drive can transmit large torque in a small size. The integration of mechanical elements and electromagnetic ones makes control easier, the motor also assemble with the decelerator. So the toroidal motor is suitable for fields such as aviation and space flight[1-3]. The servo system can be substituted by toroidal motor to simplify the structure of an existing electromechanical system. The motor can also be used in robots and other fields that require accurate control. As more and more electrical and control techniques are utilized in engineering field, new-style motors become advancing edge of electromechanical science[4,5]. Thus the dual-stator toroidal motor with hybrid excitation has more expansive application prospect.

Figure 1 shows the diagram of the dual-stator toroidal motor. The motor consists of four basic elements: (1) the worm inner stator; (2) radially positioned planets; (3) outer stator; and (4) rotor upon which the planets are mounted. Among them, the inner stator is fixed and the armature coils are mounted in helical slots of its surface. The planets adopt permanent magnets for excitation, the $\mathrm{N}$ and $\mathrm{S}$ pole permanent magnets mounted alternately on each planet. Outer stator has several helical magnetic steel or alternating NS pole permanent magnets embedded in the bracket.

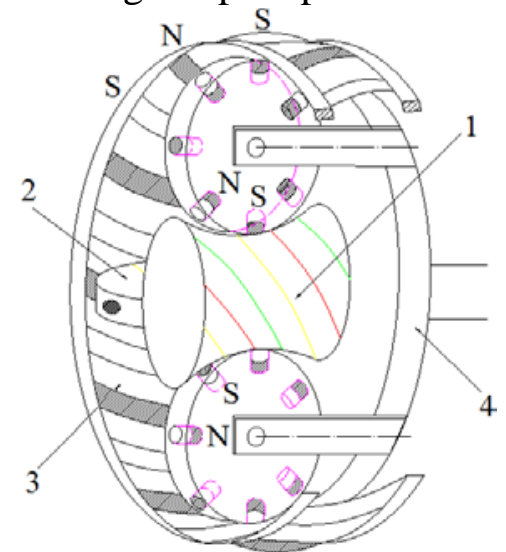

Fig.1. Diagram of toroidal motor

When a specific relationship is satisfied between the planet pitch, lead angle on the stators and the number of pole pairs, then the $\mathrm{N}$ pole of one element will corresponding to $\mathrm{S}$ pole of the other elements. A toroidal circular electromagnetic field is formed when the alternating current is connected to the coils of the armature worm. The magnetic forces between $\mathrm{N}$ and $\mathrm{S}$ poles of 
different elements are the driving force. It drives the planets to rotate about their own axes, and at the same time, the attractive force outside between planets and helical outer stator cause the rotor to rotate about its own axis. So output with a large torque at low speed is generated. In this study, from the electromechanical coupled model of toroidal motor, the transfer function of the speed control for the drive system is derived. In order to improve the speed response of the motor system, a speed feedback control model is presented and a anticipatory controller is designed. The time-domain response of speed control system is simulated and investigated. The results are useful for designing and manufacturing the controller for the novel motor.

\section{Mathematic Model of the System}

The Electromechanical toroidal system considered in this paper consists of a worm inner stator, outer stator and a rotor with planets. They couple with each other by magnetic field caused by currents and magnetic teeth. The coupled model of the motor is shown in Figure 2.

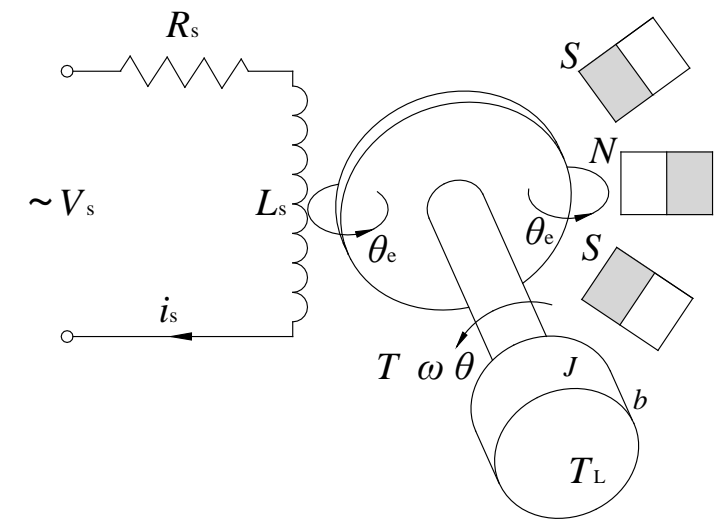

Fig.2. Electromechanical coupled model

The voltage differential equation of the worm coil is as follow

$$
v_{s}=R_{s} i_{s}+\frac{d \psi_{s}}{d t}
$$

Where $R_{\mathrm{S}}$ and $i_{\mathrm{S}}$ are resistance and current of one phase worm coils respectively. The magnetic linkage $\psi_{s}=1.5 L_{s} i_{s}+\psi_{f} \sin \theta_{e}, L_{s}$ is the average value of fundamental wave self-inductance of the worm coils, $\psi_{\mathrm{f}}$ is mutual- inductance magnetic linkage between planet teeth and the worm coil, $\theta_{e}$ which is the autorotation electric angle of planet has the transmission relationship $\theta_{\mathrm{e}}=Z_{0} \theta / 2, Z_{0}$ is tooth number of outer stator, $\theta$ is output rotating angle of the electromechanical toroidal system.

Substituting magnetic linkage into the voltage differential equation, yields

$$
v_{s}=1.5 L_{s} i_{s}+R_{s} i_{s}+Z_{0} \psi_{s} \omega \cos \left(Z_{0} \theta / 2\right) / 2
$$

The motion equation of the toroidal driven system can be given as

$$
T=K_{0} B_{r}+K_{1} i_{s}=J \ddot{\theta}+b \dot{\theta}+T_{L}
$$

Where $B_{r}$ is the remnant magnetism of permanent magnet beam of the outer stator, $K_{0}$ and $K_{1}$ are the torque constant related to the structure of the outer and inner stator respectively. $J$ and $b$ are the equivalent rotary inertia and damping coefficient of the rotor.

Combine Eq.(2) and Eq.(3), back electromotive force coefficient can be simplified as C. following equation can be obtained after Laplace transform

$$
\left\{\begin{array}{l}
V_{s}-C \omega=\left(3 L_{s 0} s / 2+R_{s}\right) I_{s} \\
K_{0} B_{r}+K_{1} I_{s}-T_{L}=(J S+b) \omega
\end{array}\right.
$$

The hybrid excitation character of toroidal motor determines that it is a two-input and single output system. The block diagram of the toroidal motor is shown in Figure 3. 


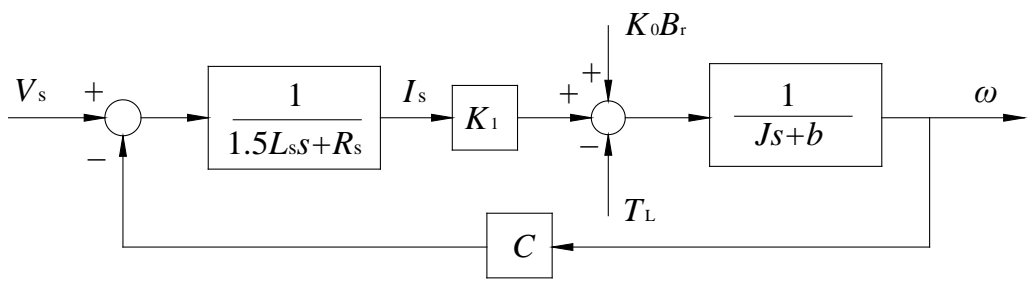

Fig.3. The block diagram of the toroidal motor

As the input of $B_{r}$ is not controllable, in order to improve the output performance of the motor, $v_{\mathrm{s}}$ is usually chosen as controllable input. From Eq.(4), the speed transfer function is given as

$$
G_{s}=\frac{K_{1}}{J L_{s} s^{2}+\left(L_{s} b+J R_{s}\right) s+\left(R_{s} b+C K_{1}\right)}
$$

\section{Speed Anticipatory Control}

The phase-lead correction can improve the response speed of the system, and meanwhile the other characteristics of the system will not be deteriorated because of the controller. The structure of the control system for toroidal motor is refined by a speed feedback loop. The transfer functions of the motor system and anticipatory controller are in the loop. The transfer function of the controller uses control algorithm as

$$
G_{c}=K_{c} \alpha \frac{T s+1}{s(\alpha T s+1)}
$$

The controller contains classical PI control algorithm which used to improve the speed control. According to the need of static speed-error constant $K_{v}$, it is known

$$
K_{v}=\lim _{s \rightarrow 0} s G_{c} G_{s}=K_{c} \alpha \frac{K_{1}}{R_{s} b+C K_{1}}
$$

In order to meet the requirement of phase margin $\gamma$, the compensator device need provide lead phase $\gamma-\gamma_{0}$, here $\gamma_{0}$ is the phase margin of system $K_{c} \alpha G_{s}$. The introducing of the other part of $G_{c}$ would cause the change the amplitude and hysteretic gain frequency in the bode diagram, so the additional phase is required, that is the phase correction unit must offer margin $\phi_{m}=\gamma-\gamma_{0}+5^{\circ}$. The equation that attenuation factor $\alpha$ need to satisfy is given as follow

$$
\frac{1-\alpha}{1+\alpha}=\sin \phi_{m}
$$

The phase margin occurs in geometrical center of the two corner frequencies $\omega_{1}=1 / T$ and $\omega_{2}=1 /(\alpha T)$ of system $K_{c} \alpha G_{s}$, that is $\omega=1 /(\sqrt{\alpha} T)$, the amplitude at this point can be calculated as

$$
\left|\frac{j \omega T+1}{j \omega \alpha T+1}\right|_{\omega=1 /(\sqrt{\alpha} T)}=\frac{1}{\sqrt{\alpha}}
$$

From the bode diagram, the frequency $\omega_{\mathrm{c}}$ corresponding to $-1 / \sqrt{\alpha}$ can be selected as the new crossover frequency, so it is reasonable to obtain $\omega_{\mathrm{c}}=\omega, T$ can be obtained as follow

$$
T=\frac{1}{\sqrt{\alpha} \omega_{c}}
$$

Combining Eq.(7) and (8), $K_{c}$ is given as

$$
K_{c}=\frac{K_{v}\left(B_{L} R+C K_{1}\right)\left(1+\sin \phi_{m}\right)}{K_{1}\left(1-\sin \phi_{m}\right)}
$$

Hence, the system with speed anticipatory control is designed, the open loop transfer function and bode diagram of the system can be obtained so as to verify whether the expected performance requirements is satisfied. Further, the response characteristics of the closed-loop system can be simulated and analyzed. 


\section{Results and Discussion}

The anticipatory controller's parameters are solved based on aforementioned equations by using a software tool developed in a Matlab environment, the system with controller is obtained. Under unit step excitation, the response simulation of the closed-loop drive system can be carried out when the controller is applied. Figure 4 shows the simulation result of the speed control system.

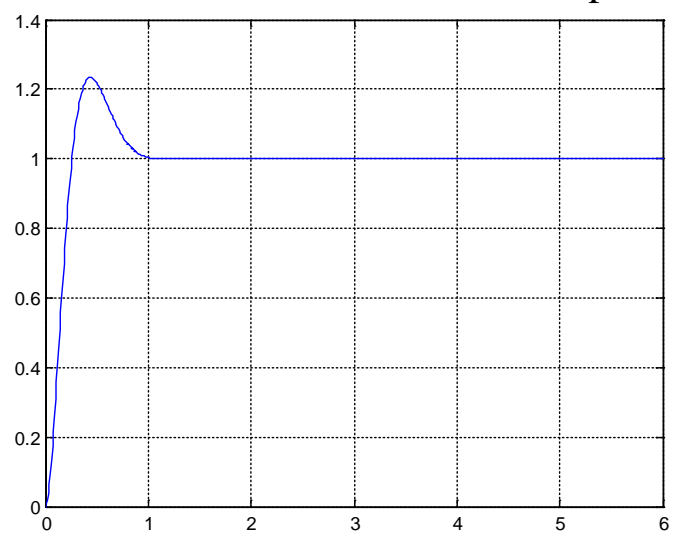

Fig.4. The simulation of the speed control system

In Fig.4, the speed closed-loop step response curve shows good regulating characteristic of the system under anticipatory controller, there is only one overshoot in the transient process. Compared with the response of the motor system, the response speed and steady-state error have been greatly improved simultaneously. From the simulation result, it is obvious that the anticipatory controller solves the problems existing in the toroidal motor well. Meanwhile, the parametrization design method is precise and easy to control, the anticipatory controller is easy to obtain so long as the controlled device and performance index are provided.

\section{Conclusion}

Based on the electromechanical coupled model of dual-stator toroidal motor system, the mathematic expression of the system was derived. The block diagram of the novel motor was presented, and a speed feedback control scheme was obtained. The parameters of anticipatory controller were solved, and the simulation result shows that a rapid response is achieved and the steady-state error is eliminated. These research results are meaningful for further research and practical application of dual-stator toroidal motor.

\section{Acknowledgement}

This project is supported by the National Natural Science Foundation of China (No.51207107).

\section{References}

[1] M.R.Kuehnle. Toroidal transmission and method and apparatus for making and assembling same[P]. United States Patent 5863273, 1999.

[2] L. Xu, Z. Huang, Contact Stresses for Toroidal drive[J]. Journal of Mechanical Design. Transactions of the ASME. 2003,125:165-168.

[3] Lizhong $\mathrm{Xu}$, Jin Huang. Torques for electromechanical integrating toroidal drive[J]. Proc. IMechE Part C Journal of Mechanical Engineering Science.2005, 219(8): 801-811.

[4] MO Huichen. Parameter Analysis on Permanent-Magnet AC Servomotors[J]. Micro machine. 2005,38: 3-6.

[5] Nedjar B, Hlioui S, Amara Y, et al. A new parallel double excitation synchronous machine[J]. IEEE Trans on Magnetics.2011, 47(9): 2252-2260. 